\title{
PCR effects of melting temperature adjustment of individual primers in degenerate primer pools
}

\author{
Ankur Naqib ${ }^{1}$, Trisha Jeon ${ }^{1}$, Kevin Kunstman ${ }^{1}$, Weihua Wang ${ }^{1}$, Yiding Shen ${ }^{1}$, Dagmar Sweeney ${ }^{1}$, \\ Marieta Hyde $^{1}$, Stefan J Green ${ }^{\text {Corresp. }}{ }^{1}$ \\ ${ }^{1}$ Sequencing Core, Research Resources Center, University of Illinois at Chicago, Chicago, IL, United States \\ Corresponding Author: Stefan J Green \\ Email address: GreenDNA@uic.edu
}

Deep sequencing of small subunit ribosomal RNA (SSU rRNA) gene amplicons continues to be the most common approach for characterization of complex microbial communities. PCR amplifications of conserved regions of SSU rRNA genes often employ degenerate pools of primers to enable targeting of a broad spectrum of organisms. One little noticed feature of such degenerate primer sets is the potential for a wide range of melting temperatures between the primer variants. The melting temperature variation of primers in a degenerate pool could lead to variable amplification efficiencies and PCR bias. Thus, we sought to adjust the melting temperature of each primer variant individually. Individual primer modifications were used to reduce theoretical melting temperature variation between primers, as well as to introduce inter-cluster nucleotide diversity during Illumina sequencing of primer regions. We demonstrate here the suitability of such primers for microbial community analysis. However, no substantial differences in microbial community structure were revealed when using primers with adjusted melting temperatures, though the optimal annealing temperature decreased. 
1 PCR effects of melting temperature adjustment of individual primers in degenerate primer

2 pools

3

4 Running title: Primer melting temperature adjustment

5

6 Ankur Naqib ${ }^{1}$, Trisha Jeon ${ }^{1}$, Kevin Kunstman ${ }^{1}$, Weihua Wang ${ }^{1}$, Yiding Shen ${ }^{1}$, Dagmar

7 Sweeney ${ }^{1}$, Marieta Hyde ${ }^{1}$, Stefan J. Green ${ }^{1 *}$

8

9 Author affiliations: ${ }^{1}$ Sequencing Core, Research Resources Center, University of Illinois at

10 Chicago, Chicago, IL, 60612, USA;

11

12 Corresponding author: Stefan J. Green, 835 S. Wolcott, A-310, Chicago, Illinois, 60612

13 Email: GreenDNA@uic.edu 


\section{Abstract}

15 Deep sequencing of small subunit ribosomal RNA (SSU rRNA) gene amplicons continues to be 16 the most common approach for characterization of complex microbial communities. PCR

17 amplifications of conserved regions of SSU rRNA genes often employ degenerate pools of

18 primers to enable targeting of a broad spectrum of organisms. One little noticed feature of such

19 degenerate primer sets is the potential for a wide range of melting temperatures between the

20 primer variants. The melting temperature variation of primers in a degenerate pool could lead to

21 variable amplification efficiencies and PCR bias. Thus, we sought to adjust the melting

22 temperature of each primer variant individually. Individual primer modifications were used to

23 reduce theoretical melting temperature variation between primers, as well as to introduce inter-

24 cluster nucleotide diversity during Illumina sequencing of primer regions. We demonstrate here

25 the suitability of such primers for microbial community analysis. However, no substantial

26 differences in microbial community structure were revealed when using primers with adjusted

27 melting temperatures, though the optimal annealing temperature decreased. 


\section{Introduction}

In molecular surveys performed in the field of molecular microbial ecology, genes such as those encoding for the small subunit ribosomal RNA (SSU rRNA), dissimilatory sulfite reductase, nitrite reductase, and more are frequently targeted to survey the total microbial community or members of specific metabolic groups. For broad surveys of microbial community structure, the SSU rRNA gene is most frequently targeted. One common feature of primers used for microbial community surveys is sequence degeneracy; that is, rather than a single primer, a mixture of multiple highly similar primers, targeting multiple variants of priming regions, is employed to cover as broad a taxonomic range of organisms as possible. Over time, the degeneracy of commonly used primers tends to increase due to the availability of additional sequence data demonstrating mismatches between primers and novel sequences. In many cases, introduction of new variants has been highly successful, allowing the detection of specific microbial clades. For example, the commonly used $16 \mathrm{~S}$ rRNA gene primer $27 \mathrm{~F}$ does not properly amplify genomic DNA from bacteria of the genus Bifidobacterium and other taxa [1, 2]. A series of additional degeneracies were introduced to improve taxonomic coverage [2]. More recently, additional degeneracies were added to the 515F primer to allow targeting of Crenarchaeota and Thaumarchaeota and to 806R to allow targeting of the SAR11 clade [3, 4].

As new variants are introduced into primer pools, primer melting temperature is rarely considered. Thus, the melting temperature $(\mathrm{Tm})$ of primer variants within a degenerate pool can have a substantial range. For example, the original ' $806 \mathrm{R}$ ' degenerate primer employed by the Earth Microbiome Project (EMP) is 18 -fold degenerate, with a theoretical Tm range of approximately $7^{\circ} \mathrm{C}$. We previously demonstrated that the annealing temperature of PCRs altered the profile of primers in a degenerate pool annealing to genomic DNA (so-called "primer 
51 utilization profiles" or PUP; [5]). The variable melting temperature of the individual primers in a

52 degenerate pool creates the potential for additional bias, as differences in free energy binding

53 between primers and perfectly matching templates will vary. Furthermore, mismatch interactions

54 could be favored when primers with high theoretical Tm values are used in PCR reactions run at

55 low annealing temperatures necessary for low Tm primers. Alternatively, some primers may not

56 have the opportunity to anneal in PCR reactions with elevated annealing temperatures. Such

57 reactions can still yield amplification due to the presence of high Tm primers in the degenerate

58 pool.

59 The objective of this study was to develop and test an experimental system to determine whether

60 the broad range of primer melting temperatures in degenerate primer pools contributes

61 substantially to observed microbial community profiles generated from amplicon-based

62 sequencing approaches. To do so, we altered each primer independently by removing nucleotides

63 from both 5' and 3' ends of oligonucleotide primers to minimize variance in primer theoretical

64 Tm. These oligonucleotides were synthesized independently and pooled in equimolar

65 concentration. Subsequently, these primer pools were used for PCR amplification of several

66 complex genomic DNA samples, followed by high-throughput sequencing. Sequencing results

67 were compared to amplicon sequence data generated from PCRs employing standard degenerate

68 primers. As a secondary objective, we sought to determine if we could modify primer sequences

69 at the 5' end to introduce nucleotide diversity into amplicons sequenced on next-generation

70 sequencers.

\section{Materials and Methods}


The most recent Earth Microbiome Project (EMP) primers, 515F (Parada) [“515F”] and 806R (Apprill) ["806R"], were utilized as the default primer set [3, 4, 6, 7]. These primers are 4-fold degenerate $(515 \mathrm{~F})$ and 24 -fold degenerate $(806 \mathrm{R})$, with theoretical melting temperatures ranging from 66.9 to $71.8^{\circ} \mathrm{C}(515 \mathrm{~F})$ and from 54.7 to $61.7^{\circ} \mathrm{C}(806 \mathrm{R})$ (Table S1). Primer theoretical melting temperatures were calculated using the OligoAnalyzer3.1 calculator [8], assuming 250

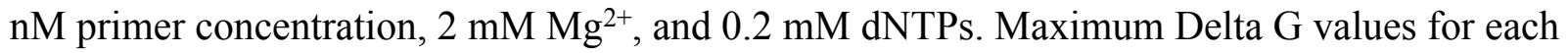
sequence were calculated using the self-dimer option in the OligoAnalyzer software. Primers were synthesized either as single degenerate primer pools (standard approach), or as individual primers without degeneracies by Integrated DNA Technologies (IDT; Coralville, IA). All primers were synthesized as LabReady and delivered at a fixed concentration of 100 micromolar. Most primers contained common sequence linkers (CS1 and CS2) at the 5' ends, as shown in Table S2. These linker sequences are necessary for the later incorporation of Illumina sequencing adapters and sample-specific barcodes. For new primer pools containing shortened primers, a combination of either 4 (" $515 \mathrm{~F}$ ") or 24 (“806R") non-degenerate primers were combined in equal volume to generate degenerate pools at 100 micromolar concentration. These primer pools were named " $515 \mathrm{~F} \_$pool" and "806R_pool”, and when these primers were used together, the primer set was named "ShortEMP". One experiment, described below, employed ShortEMP primer pools without common sequences ("NoLinker_ShortEMP") to assess the effect of the linker sequences on analysis of microbial community structure. A final set of primers, employing variable length spacers, was generated and named the "LongEMP" primer set. These primers included variable length spacers between the common sequence linkers and the gene-specific EMP primer regions, as described previously [9]. Sequencing with these 
96 primers was performed on both MiniSeq and MiSeq instruments, but data from the MiniSeq

97 using the "LongEMP" primer set did not properly merge due to read-length limitations, and was

98 not further analyzed.

Genomic DNA Templates

101 Four microbial genomic DNA (gDNA) samples were employed in this study. These include the

102 ZymoBIOMICS Microbial Community DNA standard (D6306; Zymo Research, Irvine, CA;

103 'Zymo'), as well as three environmental samples derived from Lake Michigan sediment

104 ('LMC'), garden soil ('Soil') and mammalian (rat) feces ('Feces'). Sediment, soil and fecal

105 samples were extracted using a PowerSoil DNA extraction kit (Qiagen, Hilden, Germany),

106 following the manufacturer's protocol.

A two-stage PCR amplification strategy was used to generate sequencer-ready amplicons [10].

Genomic DNA was first PCR amplified with primer set EMP (CS1_806R and CS2_515F),

ShortEMP (CS1_806R_pool and CS2_515F_pool), or LongEMP (CS1_806R_long_pool and

CS2_806R_long_pool) (Table S2). All primers contained 5' common sequence tags (known as common sequence 1 and 2, CS1 and CS2) as described previously [11]. First stage PCR amplifications were performed in 10 microliter reactions in 96-well plates, using MyTaq HS 2X mastermix (Bioline, Taunton, MA). PCR conditions were $95^{\circ} \mathrm{C}$ for 5 minutes, followed by 28

116 cycles of $95^{\circ} \mathrm{C}$ for 30 ”, variable annealing temperature for $60^{\prime \prime}$ and $72^{\circ} \mathrm{C}$ for $90^{\prime}$. For

117 temperature gradients, annealing temperatures of $40^{\circ}, 45^{\circ}$ and $50^{\circ} \mathrm{C}$ were employed. 
118 Subsequently, annealing temperatures of $45^{\circ} \mathrm{C}$ were used for ShortEMP reactions and $50^{\circ} \mathrm{C}$ for 119 EMP reactions.

120 Second stage PCR amplifications were performed in 10 microliter reactions in 96-well plates. A 121 mastermix for the entire plate was made using the MyTaq HS 2X mastermix. Each well received 122 a separate primer pair with a unique 10-base barcode, obtained from the Access Array Barcode 123 Library for Illumina (Fluidigm, South San Francisco, CA; Item\# 100-4876). These AccessArray

124 primers contained the CS1 and CS2 linkers at the 3' ends of the oligonucleotides. Cycling 125 conditions were as follows: $95^{\circ} \mathrm{C}$ for 5 minutes, followed by 8 cycles of $95^{\circ} \mathrm{C}$ for $30^{\prime \prime}, 60^{\circ} \mathrm{C}$ for 12630 " and $72^{\circ} \mathrm{C}$ for 30 ".

127 In an experiment using the NoLinker_ShortEMP primers, the above protocol was modified 128 slightly. To assess the effect of common sequence linkers on the observed microbial community 129 structure, we performed the first stage PCR amplification with NoLinker_ShortEMP primers. 130 PCR conditions were identical to those described above. During the second stage PCR amplification, both Fluidigm Access Array Barcode and the ShortEMP primers were included, and 12 cycles of amplification were performed in place of 8. In this approach, common sequences are incorporated during the second stage PCR as the ShortEMP primers with linkers amplify the NoLinker_ShortEMP amplicons; subsequently, the Fluidigm Access Array barcode primers amplify amplicons containing the common sequence linkers.

In all experiments, samples were pooled using an EpMotion5075 liquid handling robot protocol (0.6X, vol/vol; Agencourt, Beckmann-Coulter) to remove fragments smaller than 300 bp. The pooled libraries, with either a $1 \%$ or $20 \%$ phiX spike-in, were loaded onto either an 140 Illumina MiniSeq mid-output kit (2x153 paired-end reads) or an Illumina MiSeq V2 kit (2x250 
141 paired-end reads), as indicated in Table S3. Fluidigm sequencing primers, targeting the CS1 and

142 CS2 linker regions, were used to initiate sequencing. De-multiplexing of reads was performed on

143 instrument. Library preparation, pooling, and sequencing were performed at the University of

144 Illinois at Chicago Sequencing Core (UICSQC).

Bioinformatic Analysis of Sequence Data

147 Raw FASTQ files were downloaded from Illumina Basespace. Sequence reads were merged using PEAR (Paired-End Read Merger) [12] with default parameters. Merged reads were quality trimmed ( $<$ Q20 discarded) and length trimmed ( $<250$ bases were removed), and primer sequences were removed using the 'Trim reads' algorithm within the software package CLC Genomics Workbench (v11; Qiagen; Hilden, Germany). Trimmed sequences were reverse complemented using a QIIME script. Chimeras were removed using the USEARCH81 algorithm [13]. Subsequently, for environmental samples (LMC, Soil, Feces), sequences were pooled, renamed and clustered into operational taxonomic units (OTUs) at a threshold of $97 \%$ similarity (QIIME v1.8.0; [14]). Each OTU was annotated taxonomically based on the representative sequences using the UCLUST algorithm and the greengenes 13_8 reference database [15]. A biological observational matrix (BIOM) was generated from the clustered OTU data and the taxonomy data [16]. For subsequent analyses, data were rarefied using the vegan package within the R programming language. Rarefaction depths were adjusted by analysis; depth of rarefaction for each figure is shown in Table S3. The BIOMs were analyzed and visualized using the 161 software package Primer7 [17] and in the R programming environment [18]. Dendrogram creation and SIMPROF tests were conducted within Primer7. The vegan package [19] was used to generate alpha diversity indices and to calculate pairwise Bray-Curtis dissimilarity scores. 
164 Analysis of similarity (ANOSIM) calculations were performed at the taxonomic level of genus,

165 using square root transformed data. Metric multi-dimensional scaling (mMDS) plots were

166 created using the cmdscale and ggplot2 functions within R. Ellipses, representing a 95\%

167 confidence interval around group centroids, were created assuming a multivariate t-distribution

168 [20]. Taxon-level differential abundances between sample groups were identified using the

169 software package STAMP [21] employing White's non-parametric t-test [22]. P values were

170 adjusted using the Benjamini-Hochberg False Discovery Rate correction [23].

171 Sequence data from 'Zymo' samples was processed using the same pipeline, but data were not

172 clustered. Instead, merged, trimmed and chimera-cleaned data were mapped against reference

173 gene sequences for the eight bacterial reference organisms using the software package CLC

174 genomics workbench v10 (Qiagen, Aarhus, Denmark). The eight reference organisms included:

175 Lactobacillus fermentum (AJ575812), Bacillus subtilis (DQ993674), Escherichia coli (J01859),

176 Enterococcus faecalis (EU887827), Salmonella enterica (JQ694167), Listeria monocytogenes

177 (M58822), Pseudomonas aeruginosa (LN874213), and Staphylococcus aureus (L37597).

178 Mapping data were then converted to biological observation matrices for use in visualization and 179 statistical analyses, as described above. Ideal scores were calculated according to the formula

180 described previously [5]. Briefly, the Ideal score is a summation of the absolute difference

181 between the expected relative abundance and the observed relative abundance for each feature in

182 a multi-feature dataset.

Data sharing 
185 Raw sequence data files were submitted in the Sequence Read Archive (SRA) of the National

186 Center for Biotechnology Information (NCBI). The BioProject identifier for the samples is

187 PRJNA492144. Full metadata for each sample are provided in Table S3.

\section{Results}

\section{Primer design}

191

192

193

194

195

196

197

198

199

200

201

202

203

204

205

206

The design of new primers with lower variation in melting temperature was performed with two constraints, including: (a) no primer shorter than 16 bases, and (b) all degenerate positions are retained. Outside of these constraints, we aimed to minimize theoretical melting temperature variation within each degenerate primer pool. The high overall GC content and 3' run of A or T bases in the $515 \mathrm{~F}$ primer limited our ability to adjust $\mathrm{Tm}$ of this primer while meeting the above constraints. An underlying assumption of the primer modification was that shortening the primers could only increase the range of potential targets [24]. To adjust primer Tm, bases were sequentially removed from either or both 5' and 3' ends of each oligonucleotide (Figure 1a;

Table S1). The final primer design ("ShortEMP”) yielded pools of primers with overall lower average theoretical melting temperatures, and with smaller range in Tm between all primers within the pool compared to the original pool ("EMP”) (Figure 1b; Table S1). The 515F primer pool, comprised of 4 primers, was only modestly affected by the primer alterations. The Tm range prior to modification was 66.9 to $71.8^{\circ} \mathrm{C}$; after modification, the Tm range was 66.9 to $69.1^{\circ} \mathrm{C}$. The Tm range of the modified primer pool was not significantly different from that of the original pool [Wilcoxon-Mann-Whitney (WMW) test; $p=0.37$ ]. The 806R primer pool, comprised of 24 primers was more strongly affected by the alterations. The Tm range prior to 
207 modification was 54.7 to $61.7^{\circ} \mathrm{C}$; after modification, the $\mathrm{Tm}$ range was 53.6 to $56.6^{\circ} \mathrm{C}$. The $\mathrm{Tm}$

208 range of the modified primer pool was significantly different from that of the original pool

209 (WMW test; $\mathrm{p}<0.00001$ ).

210 A secondary aim of design was to introduce nucleotide diversity at the 5 ' end of the combined

211 oligonucleotides by preferential removal of bases as the 5' end of the gene-specific portion of

212 each primer (Figure 1a; Tables S1, S4, S5). Illumina's technical notes indicate that having

213 relatively even proportions of all four nucleotides during each sequencing cycle is necessary for

214 proper sequencing. This is particularly true during the first cycles of sequencing, when the

215 sequencer is still training itself to identify clusters. To improve sequence quality of so-called

216 "low diversity" libraries (e.g., 16S rRNA gene amplicon libraries), exogenous spike-ins of

217 shotgun DNA libraries derived from the virus phiX174 are typically employed. Elsewhere,

218 Lundberg et al. [9] introduced nucleotide diversity through the synthesis of primers containing

219 frameshift nucleotides. Briefly, a mixture of six different forward and six different reverse

220 primers were pooled together [9]. Each primer variant contained an identical gene-specific

221 degenerate primer at the 3' end of the oligonucleotides, but a variable number of nucleotides

222 upstream of the gene-specific portion of the primers. This variable number of upstream

223 nucleotides introduces artificial diversity into the sequencing reaction due to an effective frame

224 shift by offsetting the start of highly conserved regions of the amplicon by 1-5 bases. We aimed

225 to achieve the same effect by removing bases from the 5' end of the gene-specific portion of the

226 primers, thereby introducing the frame-shift effect (Figure 1a). To assess the effect of 5,

227 nucleotide removals on the overall nucleotide diversity at each position, we generated two in

228 silico calculations: (a) number of different nucleotides present at any given position across the

229 first 16 nucleotides of each primer pool, and (b) the Shannon index for nucleotide diversity at 
230

231

232

233

234

235

236

237

238

239

240

241

242

243

244

245

246

247

248

249

250

251

252

each of the first 16 nucleotides of each primer pool (Tables S4 and S5). The original primer pool design has very low nucleotide diversity, as the primers are perfectly synchronized and have only two or three degenerate positions. Thus, for the original 515F primer pool, all primer positions other than the $4^{\text {th }}$ and $9^{\text {th }}$ positions have only a single nucleotide represented. The average number of nucleotides present at any position is 1.125 , and the average Shannon index (natural $\log$ ) across the first 16 positions is 0.09 (Table S4). Conversely, after modifying the individual primers, the average nucleotide diversity across the 16 positions was 2.44 , with an average Shannon index of 0.77 . The maximum possible Shannon index value for 4 different nucleotides is 1.39. A similar effect was observed for the $806 \mathrm{R}$ primer pool (Table S5). Prior to redesign, the average nucleotide diversity across the first 16 positions was 1.38 , compared to 3.19 after redesign. Likewise, Shannon index increased from 0.2 to 1.0 after redesign.

\section{Microbiome profiling using EMP and ShortEMP primers}

To assess the effects of primer modifications, initially a single complex genomic DNA template (LMC) was profiled across an annealing temperature gradient using EMP and ShortEMP primer sets. We previously observed a strong and consistent shift in microbial community structure associated with increasing annealing temperatures from $40^{\circ} \mathrm{C}$ to $55^{\circ} \mathrm{C}$, with specific taxa such as Prevotellaceae increasing in relative abundance with increasing annealing temperature in mammalian fecal samples [5]. Therefore, annealing temperatures of $40^{\circ} \mathrm{C}, 45^{\circ} \mathrm{C}$ and $50^{\circ} \mathrm{C}$ were tested in this study. The single gDNA template was PCR amplified at each of the temperatures and primer sets with six technical replicates. The observed microbial community from the source gDNA template was significantly affected by primer set (EMP or ShortEMP) and by annealing temperature (Figure 2). Variation in microbial community structure by temperature was 
253 described primarily by the MDS axis 1 , while that of primer set was described primarily by MDS

254 axis 2. The magnitude of the shift was small, with overall Bray-Curtis similarity of all

255 comparisons between EMP and ShortEMP replicates $>0.84$ (Figure 3A). Within each primer set,

256 replicates had Bray-Curtis similarity values $>0.89$, with ShortEMP replicates having slightly and

257 significantly greater similarity (Figure S1, LMC). No significant effect of primer set and

258 annealing temperature on alpha diversity indices (e.g., richness and Shannon index) at the genus-

259 or OTU-level were observed for LMC (Table 1). At each annealing temperature, relatively few

260 taxa (16-28 genus-level taxa) were significantly differently abundant between the EMP and

261 ShortEMP analyses (Figure 2B, S3).

262 Based on the results of the temperature gradient analysis (above) and testing of a mock

263 community standard (below), an annealing temperature of $45^{\circ} \mathrm{C}$ was chosen for additional

264 analyses employing the ShortEMP primers and $50^{\circ} \mathrm{C}$ for EMP primers. Two additional complex

265 microbial samples were analyzed at these temperatures, with six technical replicates for each.

266 These samples include 'feces' and 'soil'. In each of these analyses, a significant effect of primer

267 set was observed (Figure 3B,C; ANOSIM R=1, p<0.008), and in each case, the magnitude of the

268 effect was similar, with overall Bray-Curtis similarity between all replicates of the same sample

$269>0.82$ (Figure 3B,C). Small effects of primer set on alpha diversity indices were observed, and

270 only the OTU-level Shannon index for the soil sample was significantly different between

271 ShortEMP primers relative to EMP primers (7.09 vs 7.16) (Table 1).

272

273 Interrogation of a mock community with EMP, ShortEMP, LongEMP and NoLinker_ShortEMP

274 primer sets 
275 The ZymoBIOMICS Microbial Community DNA standard (“Zymo”) was used as a gDNA

276 template to assess the capability of the various primer sets to characterize microbial

277 communities. The Zymo standard is composed of 8 bacterial taxa and 2 fungal taxa at varying

278 levels of abundance (fungi are not amplified by the $515 \mathrm{~F} / 806 \mathrm{R}$ primer set). When analyzed using

279 16S rRNA gene amplicon sequencing, the relative abundance of each strain should range from

$2804.2 \%$ to $18.4 \%$. Both primer sets (EMP and ShortEMP) generated highly similar but significantly

281 distinct results (Figure 3D; ANOSIM R=1, p=0.0019). For each replicate, an Ideal Score [5] was

282 calculated based on the expected relative abundance of each taxon. The Ideal Score represents a

283 summation of the difference in relative abundance of each taxon in a mock community. A perfect

284 representation of the expected relative abundance of each taxon will yield an ideal score of zero,

285 with values greater than zero representing increasing discordance from the expected results. Ideal

286 score results were generated for each of the six technical replicates and compared at annealing

287 temperatures of $40^{\circ} \mathrm{C}, 45^{\circ} \mathrm{C}$ and $50^{\circ} \mathrm{C}$ for EMP and ShortEMP primers (Table 2). When

288 employing the EMP primers for analysis of the Zymo standard, annealing temperatures below

$28950^{\circ} \mathrm{C}$ produced progressively worse results (i.e., higher ideal scores). When employing the

290 ShortEMP primers for analysis of the Zymo standard, annealing temperatures of $45^{\circ} \mathrm{C}$ and $50^{\circ} \mathrm{C}$

291 yielded similar results, with low ideal scores relative to $40^{\circ} \mathrm{C}$ (Table 2).

292 Ideal scores indicated that when used at their optimal annealing temperature EMP primers were

293 slightly, but significantly, better than ShortEMP primers in recovering the expected distribution

294 of the Zymo bacterial taxa. Although the EMP primers slightly outperformed the ShortEMP

295 primers when compared to the expected distribution of eight bacterial taxa, the ShortEMP

296 primers better tolerated a broader range of annealing temperatures. ShortEMP primers produced 
297 nearly identical results at $45^{\circ} \mathrm{C}$ and $50^{\circ} \mathrm{C}$ (Table 2), demonstrating that the lower overall $\mathrm{Tm}$ of

298 the primer pool can contribute to a shift in optimum annealing temperature.

299 We sought to assess whether the addition of spacer regions, as described previously by Lundberg 300 et al. [9], would alter the efficiency of amplification in this system. We synthesized primers 301 containing common sequence linkers, a 0-5 base frameshift sequence, a two-base 'linker', and 302 the EMP primer sequence (Table S2; 'LongEMP'). A pool of 6 forward primers and 6 reverse 303 primers were used in the standard two-stage PCR protocol to amplify the mock community 304 gDNA. The observed mock community generated from LongEMP analyses was most similar, 305 and not significantly different, from that generated using the EMP primer set (Figure 4;

306 ANOSIM R=-0.179, $\mathrm{p}=0.965$ ). EMP and LongEMP primer sets generated more similar observed 307 microbial communities, relative to the ShortEMP primer set (ANOSIM $\mathrm{R}=0.715-0.722$, $308 \mathrm{p}=0.002$ ). Although significant, the difference was not large, and EMP, ShortEMP and 309 LongEMP generated highly similar results when applied to the mock community DNA standard 310 (Figure 4).

311 We also sought to assess whether the common sequencer linkers themselves contributed to 312 distortion of the underlying microbial community structure. Conceptually, the 3' ends of the 313 linker sequences could interact with genomic DNA templates, leading to preferential 314 amplification of some templates. To avoid initial interaction of linker sequences with gDNA 315 templates, we performed the first stage PCR amplification with ShortEMP primers without 316 common sequence linkers (NoLinker_ShortEMP). After 28 cycles of amplification, the 317 generated PCR amplicons were transferred to the second stage PCR amplification, as performed 318 for all other reactions. To allow these amplicons without linkers to be prepared for Illumina 319 sequencing, both ShortEMP primers (with common sequence linkers) and Fluidigm Access 
320 Array barcoding primers were added to the second stage reaction. Final amplicons were

321 sequenced and the resulting sequence data analyzed together with EMP, ShortEMP and

322 LongEMP primer sets (Figure 4). The observed community generated by the

323 NoLinker_ShortEMP primer set was significantly different than those generated by EMP,

324 ShortEMP and LongEMP primer sets (ANOSIM R=1, $=0.002$ ), and the observed community

325 structure was also more divergent from the expected structure than those generated with the other

326 primer sets (Figure 4). Thus, the common sequence linkers do not appear to substantially alter

327 the observed mock microbial community structure, and removing the linkers leads to a more

328 complex workflow and a poorer representation of the mock community.

329 We should note that the Zymo standard is not an ideal mock community for assessing the action

330 of a degenerate primer pool. We sought to identify the sequences of the eight bacterial DNA

331 templates in the region of the $515 \mathrm{~F}$ and $806 \mathrm{R}$ primers, and despite the moderately broad range of

332 bacterial taxa included (Proteobacteria, Actinobacteria, and Firmicutes), all sequences were

333 identical at the primer annealing locations. This mock community is, therefore, appropriate for

334 determining that an overall PCR and sequencing workflow is successful but is not a good

335 approximation of a highly complex natural microbial community, where even conserved sites

336 such as the $515 \mathrm{~F}$ and $806 \mathrm{R}$ primer sites contain considerable sequence heterogeneity.

337 We also used the amplicons generated from the Zymo standard to assess variability introduced

338 by sequencing on the Illumina MiSeq or MiniSeq platforms. For the EMP, ShortEMP, and

339 NoLinker_ShortEMP, identical amplicons were sequenced and analyzed on both platforms. The

340 LongEMP amplicon reads generated on the Illumina MiniSeq did not consistently merge, and

341 were not included in the analysis. This is due to the additional 'spacer' bases (up to 6 on each

$342 \mathrm{read}$ ) and the short overlap of paired-end reads generated with $2 \times 153$ bases reads on the 
343 MiniSeq. MiSeq and MiniSeq results from the same amplicons clustered together, regardless of

344 primer set (Figure 4). For the EMP and ShortEMP primer sets, the effect of sequencing platform

345 was not significant (ANOSIM $\mathrm{R}=0.166, \mathrm{p}=0.091$ and $\mathrm{R}=0.05, \mathrm{p}=0.281$, respectively). For the

346 NoLinker_ShortEMP primer set, a significant effect of sequencing platform was observed

347 (ANOSIM R=0.416, $\mathrm{p}=0.002$ ). Thus, a slight effect of sequencing platform was observed, but

348 the choice of platform did not alter biological conclusions.

Assessing the need for phiX spike-in with ShortEMP primers

351

352

353

354

355

356

357

358

359

360

361

362

363

364

We sequenced ShortEMP amplicons on an Illumina MiniSeq run without substantial phiX spikein to determine if ShortEMP amplicons by themselves produced sufficient nucleotide diversity to allow proper clustering. These amplicons, generated from Lake Michigan sediment, at annealing temperatures of 40, 45 and 50, were analyzed together with the same amplicons generated on a MiniSeq run with a $20 \%$ phiX spike-in. The overall quality of the run was extremely high ( $>96 \%$ pass-filter, $>97 \%$ Q30 with approximately $1.75 \%$ phiX spike-in), and results were similar to those generated on a 20\% phiX run (Figure 5). Slight trends towards small, significant differences were observed between the two sequencing runs, but the overall magnitude of the difference was small.

\section{Discussion}

We demonstrate here that the strategy to remove nucleotides from the 5' ends of individual primers is effective for introducing nucleotide diversity into a primer pool, and for reducing the Tm range of the primers within a degenerate primer pool. Selective removal of bases at the 3' 
365 end can be used together with 5' base removal to adjust overall primer melting temperature. Base

366 removal at the 3' end, when using the Fluidigm sequencing protocol, does not impact nucleotide

367 diversity for the purposes of Illumina sequencing. In this study, to ensure that the greatest

368 nucleotide diversity was present during the initial cycles of the first sequencing reaction, the

369 'CS1' linker sequence was attached to the 806R primer. This approach is inverted compared to

370 standard EMP workflows, where the CS1 linker is attached to the 515F primer [10]. For future

371 designs, the choice of linker can be adjusted as needed to ensure the highest nucleotide diversity

372 during the initial cycles of the first read of Illumina sequencers, when cluster identification is

373 performed. When amplicons generated with the modified primers were sequenced on an Illumina

374 MiniSeq sequencer with $<2 \%$ phiX, the data quality and community analyses were consistent

375 with the same amplicons generated on a run with $20 \%$ phiX. Therefore, we conclude that the

376 nucleotide diversity created by removing bases at the 5 ' ends of primers to effect the frameshift

377 was sufficient to allow for sequencing without substantial phiX inclusion.

378 We sought to determine whether the broad range of primer melting temperatures in degenerate

379 primer pools contributes substantially to observed microbial community profiles. Individual

380 primer modifications were used to reduce theoretical melting temperature variation between

381 primers with a degenerate pool, and the modified primers were used to amplify mock and

382 environmental samples. When compared to amplicons generated using standard primer sets, the

383 reducing melting temperature variability in the modified primer pools did not substantially alter

384 observed microbial community structure in the tested samples. The modified primers had limited

385 or no effect on measured alpha diversity in complex microbial samples and shifts in microbial

386 community structure associated with the modified primers relative to the standard primers were

387 small in scale. The shortened primers appear to have greater tolerance for lower annealing 
388 temperatures than the standard primers. We considered the possibility that high Tm primers 389 could dominate primer-template interactions, leading to variable amplification efficiencies in 390 PCR and reduced taxonomic coverage. Our results, however, do not support any substantial 391 modification in the target range for the modified primers, even at low annealing temperatures.

392 We do note, however, that the 515F and 806R primers themselves have highly divergent

393 theoretical melting temperatures, and that this discrepancy could contribute to the difficulty in 394 expanding the targeted taxonomic range of PCR-based microbiome sequencing.

\section{Conclusions}

397 We demonstrate here a novel method to introduce nucleotide diversity into PCR amplicons for sequencing on Illumina sequencers. Through selective removal of bases at the 5 ' end of oligonucleotide primers, nucleotide diversity can be introduced without substantial effect on the activity of the primers themselves. When employing this strategy with a commonly-used primer set targeting microbial SSU rRNA genes, no substantial effects on observed microbial community structures were observed.

\section{Acknowledgements}

We kindly thank Maryam Elfeki and Brian Murphy for the supply of lake sediment genomic 406 DNA and Katherine Mak for the supply of rat fecal genomic DNA. 
409 Figure 1: Schematic of primer design and primer theoretical melting temperature

410 distribution. (A) Standard degenerate primer pools are synchronized and have low nucleotide

411 diversity when sequenced on Illumina sequencers. Nucleotides were removed from the 5' ends of

412 locus-specific portions of oligonucleotide primers to adjust melting temperature and to introduce

413 nucleotide diversity. Nucleotides were removed from the 3' ends of locus-specific portions of

414 primers to adjust melting temperature only. Sequencing reactions are initiated using the

415 'common sequences' (purple lines) adjacent to the locus-specific regions of the PCR primers. (B)

416 Distribution of theoretical melting temperatures (Tm) for primer pools using standard EMP

417 primers and modified ShortEMP primers. Modified 515F primer pool Tm distribution is not

418 significantly different from the standard 515F primer pool (Wilcoxon-Mann-Whitney test;

$419 \mathrm{p}=0.37$ ), while modified 806R primer pool Tm distribution is significantly different from the

420 standard 806R primer pool (WMW test; $\mathrm{p}<0.00001$ ).

421 Figure 2: Effect of annealing temperature and primer set on observed microbial

422 community in Lake Michigan sediment. A composite sample of gDNA from Lake Michigan

423 sediment was amplified using EMP or ShortEMP primer pools at annealing temperatures of 40,

42445 and $50^{\circ} \mathrm{C}$ and sequenced on an Illumina MiniSeq instrument. Sequence data were rarefied to a

425 depth of 17,500 sequences per sample. (A) Genus-level annotations of sequence data were

426 visualized using metric multidimensional scaling (mMDS) employing a distance matrix based on

427 Bray-Curtis similarity. Across all temperatures, the use of EMP and ShortEMP primers resulted

428 in slightly, but significantly, different observed microbial communities (ANOSIM R=0.473;

$429 \mathrm{p}<0.001$; MDS axis 2). Increasing annealing temperature also led to significant changes in

430 observed microbial community structure (ANOSIM R=0.694, $\mathrm{p}<0.001$; MDS axis 1 ). Observed

431 microbial community structures at all temperature and primer set combinations were 
432 significantly different (ANOSIM R values $0.748-1.0 ; \mathrm{p}<0.002$ ). (B) A taxon-by-taxon analysis

433 was performed to identify taxa with significantly different relative abundance between EMP and

434 ShortEMP primer sets. Shown is the comparison between EMP and ShortEMP primers at $40^{\circ} \mathrm{C}$

435 annealing temperature; comparisons at $45^{\circ} \mathrm{C}$ and $50^{\circ} \mathrm{C}$ are shown in Figure S3. Genus-level

436 annotations are shown (when available), and the mean relative abundance (six technical

437 replicates) for each primer set is shown, together with the difference in mean proportions. For

438 each comparison a q-value, calculated in the software package STAMP using White's non-

439 parametric t-test along with a Benjamini-Hochberg FDR correction, is shown. Only significantly

440 differently abundant taxa $(\mathrm{q}<0.05)$ are shown. Sequences annotated as Stramenopiles are derived

441 from SSU rRNA genes of chloroplasts from these organisms.

442 Figure 3: Effect of primer set on observed microbial communities in complex microbial

443 samples. Genus-level annotations of sequence data sequenced on an Illumina MiSeq instrument

444 were visualized using mMDS ordination employing a distance matrix based on Bray-Curtis similarity. X-axes represent MDS axis 1 and y-axes represent MDS axis 2 for all sample types.

446 Sequence data were rarefied to different depths for each sample type (Table S3). For each

447 sample, six technical replicates were performed at optimal annealing temperatures of $45^{\circ} \mathrm{C}$

448 (ShortEMP) and $50^{\circ} \mathrm{C}$ (EMP). Small, but significant, shifts in microbial communities were 449 observed between EMP and ShortEMP primers for (a) Lake Michigan sediment (LMC), 450 ANOSIM R=1, P=0.0021, (b) rat feces (Feces), ANOSIM R=1, $\mathrm{P}=0.0027$, (c) garden soil (Soil), 451 ANOSIM R=1, P=0.0033, and ZymoBIOMICS Microbial Community DNA standard (Zymo), 452 ANOSIM $\mathrm{R}=1, \mathrm{P}=0.0019$. The range of Bray-Curtis dissimilarity $(\mathrm{BCD})$ between EMP and 453 ShortEMP technical replicates is shown above each figure. Ellipses represent a 95\% confidence 454 interval around the centroid. Two outliers from the fecal analysis were removed (see Figure S2). 
455 Figure 4: Comparison of representation of mock community standard using all primer sets

456 and sequencing platforms. A single mock community standard ('Zymo') consisting of 8

457 bacterial taxa was amplified using EMP, ShortEMP, LongEMP and NoLinker_ShortEMP

458 primers using both MiniSeq and MiSeq platforms and a dendrogram representing Bray-Curtis

459 similarity is shown. Rarefaction was performed to a depth of 4,000 sequences per sample. The

460 similarity profile routine (SIMPROF) test was performed to identify clusters with non-random

461 structure. Nodes with a non-random structure (significance level of 5\%) are indicated with black

462 or colored circles. The ideal representation of the standard is shown as a pink asterisk. The

463 observed community generated using NoLinker_ShortEMP primers (node with yellow circle)

464 was significantly divergent from those observed with all other primer sets (ANOSIM R=1,

$465 \mathrm{p}=0.002$ ) and from the Zymo standard, and a significant effect of sequencing platform was

466 observed with these primers $(\mathrm{R}=0.416, \mathrm{p}=0.002,462$ permutations $)$ The microbial communities

467 observed with EMP and LongEMP primers were most similar to each other (node with blue

468 circle). No significant effect of sequencing platform was observed with EMP primers $(\mathrm{R}=0.166$,

$469 \mathrm{p}=0.091$ ) or ShortEMP primers (node with red circle; ANOSIM R=0.05, $\mathrm{p}=0.281$ ). The

470 microbial communities observed with EMP, LongEMP and ShortEMP clustered together with

471 the Zymo standard (node with green circle). No data are shown for LongEMP with the MiniSeq

472 platform, due to incomplete merging of paired-end reads.

\section{Figure 5: Effect of phiX spike-in on observed microbial communities with amplicons}

474 generated using ShortEMP primers. Genus-level annotations of sequence data generated on an

475 Illumina MiniSeq instrument were visualized using mMDS ordination employing a distance

476 matrix based on Bray-Curtis similarity. Rarefaction was performed to a depth of 30,000

477 sequences per sample. For each ShortEMP PCR condition, six technical replicates were analyzed 
478 using either $\sim 20 \%$ or $\sim 1 \%$ phiX spike-in. Small differences, consistent with run-to-run variation,

479 were observed between the two sequencing runs (ANOSIM: $40^{\circ} \mathrm{C} \mathrm{R}=0.146, \mathrm{p}=0.052 ; 45^{\circ} \mathrm{C}$

$480 \mathrm{R}=0.394, \mathrm{p}=0.011 ; 50^{\circ} \mathrm{C} \mathrm{R}=0.178, \mathrm{p}=0.100$ ). Ellipses represent a $95 \%$ confidence interval

481 around the centroid.

482

483

484

485

486

487

488

489

490

491

492

493

494

495

496

497

498

499

500

Figure S1: Box plots of within-group Bray-Curtis dissimilarity scores for microbiome analyses conducted with EMP and ShortEMP primer sets. For each sample, Bray-Curtis dissimilarity was calculated for 6 technical replicates with EMP primers (15 comparisons), and 6 technical replicates with ShortEMP primers (15 comparisons). A comparison of median withinsample similarity for replicates from EMP and ShortEMP amplifications was performed, and were significantly different for LMC, Feces and Soil (Mann-Whitney test, $\mathrm{P}<0.053$ ). An outlier of one replicate from both EMP and ShortEMP fecal analyses was removed (see Figure S2).

Figure S2: Analysis of fecal sample replicates including outliers. A single technical replicate, representing an outlier, was removed from both EMP and ShortEMP analyses (Figures 3 and S1). Analyses in this figure are shown including the deep outliers. Inclusion of the outliers does not modify the conclusions of the analysis. However, the ShortEMP outlier is greatly different from all other technical replicates from all samples in the study. (A) Genus-level annotations of sequence data were visualized using mMDS ordination employing a distance matrix based on Bray-Curtis similarity. Six technical replicates were performed at optimal annealing temperatures of $45^{\circ} \mathrm{C}$ (ShortEMP) and $50^{\circ} \mathrm{C}$ (EMP). Small, but significant, shifts in microbial communities were observed between EMP and ShortEMP primers for Feces (ANOSIM R=0.68, $\mathrm{P}=0.0025)$. Bray-Curtis dissimilarity $(\mathrm{BCD})$ between EMP and ShortEMP technical replicates is shown above the figure. Ellipses represent a 95\% confidence interval around the centroid. (B) 
501 Box plot of within-group Bray-Curtis dissimilarity scores for microbiome analyses conducted

502 with EMP and ShortEMP primer sets on fecal DNA.

503 Figure S3: Significant differences in taxon relative abundance between EMP and

504 ShortEMP analyses of Lake Michigan Sediment at varying annealing temperatures. Genus-

505 level annotations are shown (when available), and the mean relative abundance (six technical

506 replicates) for each primer set is shown, together with the difference in mean proportions. For

507 each comparison a q-value, calculated in the software package STAMP using White's non-

508 parametric t-test along with a Benjamini-Hochberg FDR correction, is shown. Only significantly

509 differently abundant taxa $(\mathrm{q}<0.05)$ are shown. Sequences annotated as Stramenopiles are derived

510 from SSU rRNA genes of chloroplasts from these organisms.

\section{Figure S4: Significant differences in taxon relative abundance between EMP and}

512 ShortEMP analyses for LMC, Feces, Soil and Zymo samples. Genus-level annotations

513 (except Zymo) are shown (when available), and the mean relative abundance (six technical

514 replicates) for each primer set is shown, together with the difference in mean proportions. For

515 each comparison a q-value, calculated in the software package STAMP using White's non-

516 parametric t-test along with a Benjamini-Hochberg FDR correction, is shown. Only significantly

517 differently abundant taxa $(\mathrm{q}<0.05)$ are shown. Zymo sequences were annotated to the taxonomic

518 level of species by mapping to known references.

519

520 Table Legends

521 Table 1: Alpha diversity indices of observed microbial communities. Shannon indices and

522 richness were calculated at the taxonomic levels of genus and at the operational taxonomic unit 
523 (OTU97) level. For each primer set (EMP or ShortEMP), an average and standard deviation of

524 six technical replicates is shown. Kruskal-Wallis tests (KW) were performed to determine if

525 observed diversity was significantly different between EMP and ShortEMP analyses. Diversity

526 indices were not significantly different between primer sets with the exception of OTU-level

527 Shannon index for the Soil sample.

528 Table 2: Ideal score calculations for analysis of the 'Zymo' Standard. The Ideal score is a

529 summation of the absolute difference between the expected relative abundance and the observed

530 relative abundance for each feature in a multi-feature dataset. Lower Ideal scores indicate a

531 better representation of the expected community. Kruskal-Wallis test (KW) p-values are shown

532 for comparisons across temperatures or between EMP and ShortEMP primers at single

533 temperatures. SD = standard deviation.

534 Table S1: Original (EMP) and modified (ShortEMP) primer variants for 515F and 806R

535 primers

536 Table S2: Primer sequences used in this study

537 Table S3: Mapping file metadata associated with all samples used in this study

538 Table S4: Nucleotide diversity calculations for EMP and ShortEMP 515F primers

539 Table S5: Nucleotide diversity calculations for EMP and ShortEMP 806R primers 
541 1. Hayashi H, Sakamoto M, Benno Y: Evaluation of three different forward primers by terminal restriction 
591 21. Parks DH, Tyson GW, Hugenholtz P, Beiko RG: STAMP: statistical analysis of taxonomic and functional

22. White JR, Nagarajan N, Pop M: Statistical Methods for Detecting Differentially Abundant Features in Clinical Metagenomic Samples. Plos Comput Biol 2009, 5(4).

23. Hochberg Y, Benjamini Y: More Powerful Procedures for Multiple Significance Testing. Stat Med 1990, 9(7):811-818.

24. Isenbarger TA, Finney M, Ríos-Velázquez C, Handelsman J, Ruvkun G: Miniprimer PCR, a new lens for viewing the microbial world. Applied environmental microbiology 2008, 74(3):840-849. 


\section{Figure $\mathbf{1}$ (on next page)}

Schematic of primer design and primer theoretical melting temperature distribution.

(A) Standard degenerate primer pools are synchronized and have low nucleotide diversity when sequenced on Illumina sequencers. Nucleotides were removed from the 5 ' ends of locus-specific portions of oligonucleotide primers to adjust melting temperature and to introduce nucleotide diversity. Nucleotides were removed from the 3' ends of locus-specific portions of primers to adjust melting temperature only. Sequencing reactions are initiated using the 'common sequences' (purple lines) adjacent to the locus-specific regions of the PCR primers. (B) Distribution of theoretical melting temperatures (Tm) for primer pools using standard EMP primers and modified ShortEMP primers. Modified 515F primer pool Tm distribution is not significantly different from the standard 515F primer pool (Wilcoxon-MannWhitney test; $p=0.37$ ), while modified $806 \mathrm{R}$ primer pool Tm distribution is significantly different from the standard 806R primer pool (WMW test; $p<0.00001$ ). 


\section{Figure 2 (on next page)}

Effect of annealing temperature and primer set on observed microbial community in Lake Michigan sediment

A composite sample of gDNA from Lake Michigan sediment was amplified using EMP or ShortEMP primer pools at annealing temperatures of 40,45 and $50^{\circ} \mathrm{C}$ and sequenced on an Illumina MiniSeq instrument. Sequence data were rarefied to a depth of 17,500 sequences per sample. (A) Genus-level annotations of sequence data were visualized using metric multidimensional scaling (mMDS) employing a distance matrix based on Bray-Curtis similarity. Across all temperatures, the use of EMP and ShortEMP primers resulted in slightly, but significantly, different observed microbial communities (ANOSIM R=0.473; $p<0.001 ;$ MDS axis 2). Increasing annealing temperature also led to significant changes in observed microbial community structure (ANOSIM $R=0.694, p<0.001$; MDS axis 1 ). Observed microbial community structures at all temperature and primer set combinations were significantly different (ANOSIM R values 0.748-1.0; $p<0.002$ ). (B) A taxon-by-taxon analysis was performed to identify taxa with significantly different relative abundance between EMP and ShortEMP primer sets. Shown is the comparison between EMP and ShortEMP primers at $40^{\circ} \mathrm{C}$ annealing temperature; comparisons at $45^{\circ} \mathrm{C}$ and $50^{\circ} \mathrm{C}$ are shown in Figure S3. Genus-level annotations are shown (when available), and the mean relative abundance (six technical replicates) for each primer set is shown, together with the difference in mean proportions. For each comparison a q-value, calculated in the software package STAMP using White's nonparametric t-test along with a Benjamini-Hochberg FDR correction, is shown. Only significantly differently abundant taxa $(q<0.05)$ are shown. Sequences annotated as Stramenopiles are derived from SSU rRNA genes of chloroplasts from these organisms. 
A

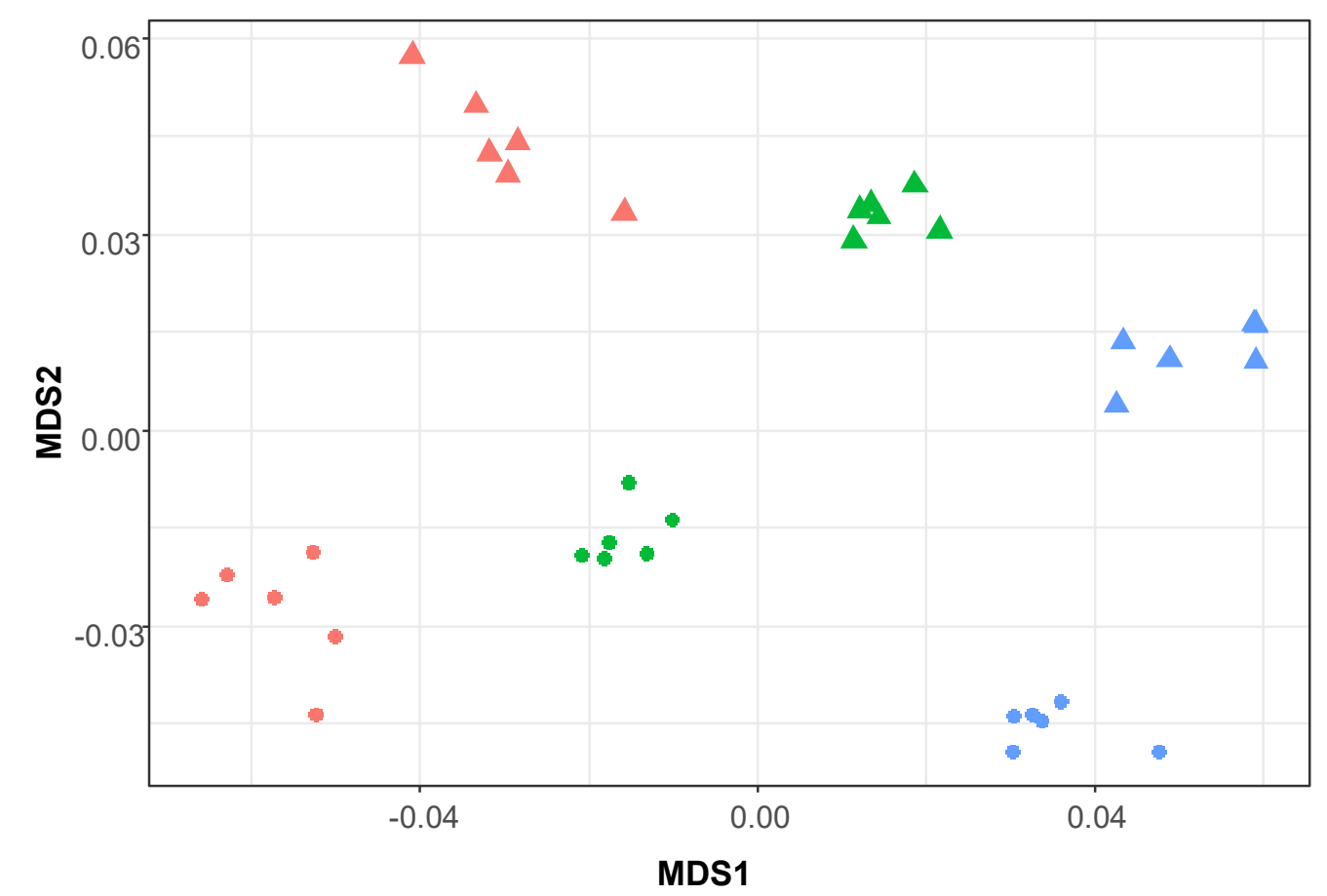

B






\section{Figure 3 (on next page)}

Effect of primer set on observed microbial communities in complex microbial samples

Genus-level annotations of sequence data sequenced on an Illumina MiSeq instrument were visualized using mMDS ordination employing a distance matrix based on Bray-Curtis similarity. X-axes represent MDS axis 1 and y-axes represent MDS axis 2 for all sample types. Sequence data were rarefied to different depths for each sample type (Table S3). For each sample, six technical replicates were performed at optimal annealing temperatures of $45^{\circ} \mathrm{C}$ (ShortEMP) and $50^{\circ} \mathrm{C}$ (EMP). Small, but significant, shifts in microbial communities were observed between EMP and ShortEMP primers for (a) Lake Michigan sediment (LMC), ANOSIM $R=1, P=0.0021$, (b) rat feces (Feces), ANOSIM $R=1, P=0.0027$, (c) garden soil (Soil), ANOSIM $\mathrm{R}=1, \mathrm{P}=0.0033$, and ZymoBIOMICS Microbial Community DNA standard (Zymo), ANOSIM $R=1, P=0.0019$. The range of Bray-Curtis dissimilarity (BCD) between EMP and ShortEMP technical replicates is shown above each figure. Ellipses represent a 95\% confidence interval around the centroid. Two outliers from the fecal analysis were removed (see Figure S2). 
A

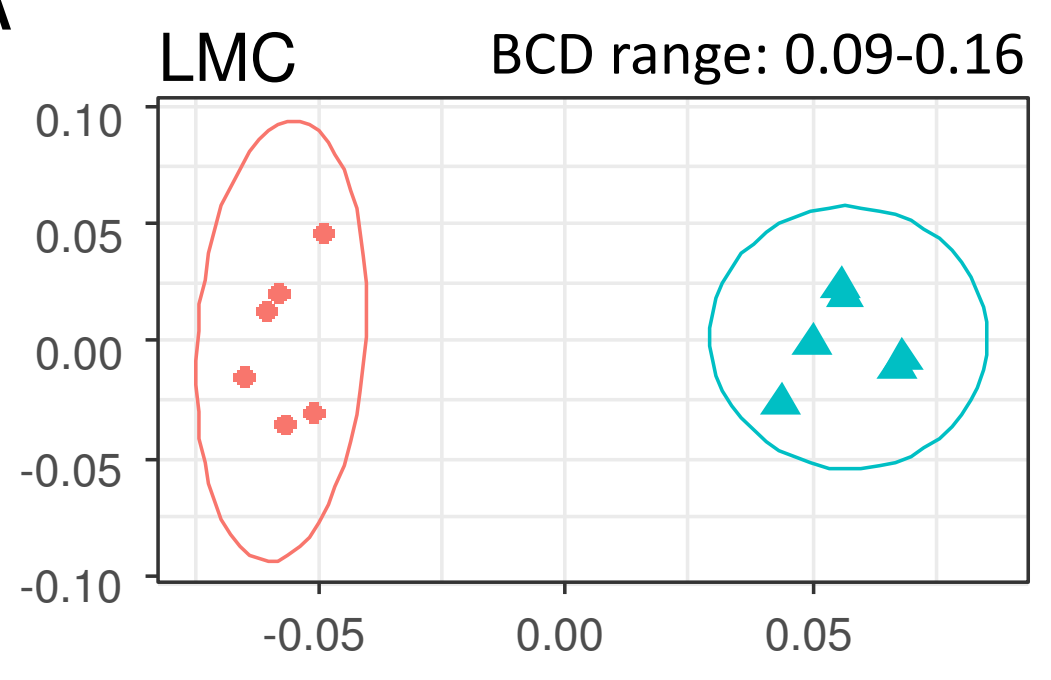

C



B

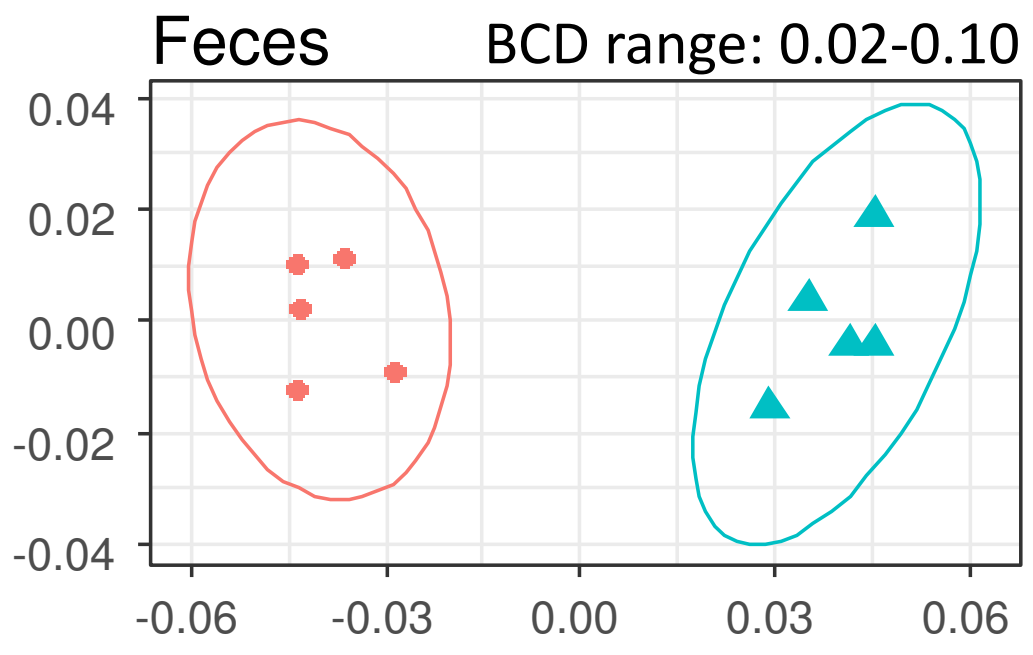

D

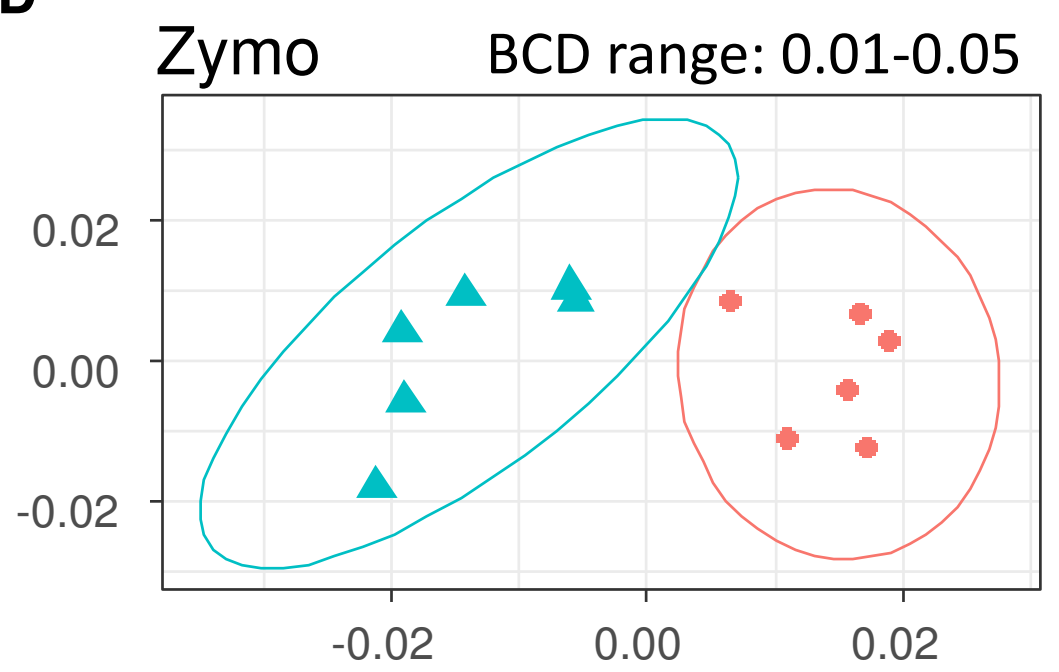

Primer

- EMP

- ShortEMP 


\section{Figure 4(on next page)}

Comparison of representation of mock community standard using all primer sets and sequencing platforms

A single mock community standard ('Zymo') consisting of 8 bacterial taxa was amplified using EMP, ShortEMP, LongEMP and NoLinker_ShortEMP primers using both MiniSeq and MiSeq platforms and a dendrogram representing Bray-Curtis similarity is shown. Rarefaction was performed to a depth of 4,000 sequences per sample. The similarity profile routine (SIMPROF) test was performed to identify clusters with non-random structure. Nodes with a non-random structure (significance level of 5\%) are indicated with black or colored circles. The ideal representation of the standard is shown as a pink asterisk. The observed community generated using NoLinker_ShortEMP primers (node with yellow circle) was significantly divergent from those observed with all other primer sets (ANOSIM $R=1$, $p=0.002)$ and from the Zymo standard, and a significant effect of sequencing platform was observed with these primers ( $R=0.416, p=0.002,462$ permutations) The microbial communities observed with EMP and LongEMP primers were most similar to each other (node with blue circle). No significant effect of sequencing platform was observed with EMP primers $(R=0.166, p=0.091)$ or ShortEMP primers (node with red circle; ANOSIM $R=0.05, p=0.281$ ). The microbial communities observed with EMP, LongEMP and ShortEMP clustered together with the Zymo standard (node with green circle). No data are shown for LongEMP with the MiniSeq platform, due to incomplete merging of paired-end reads. 


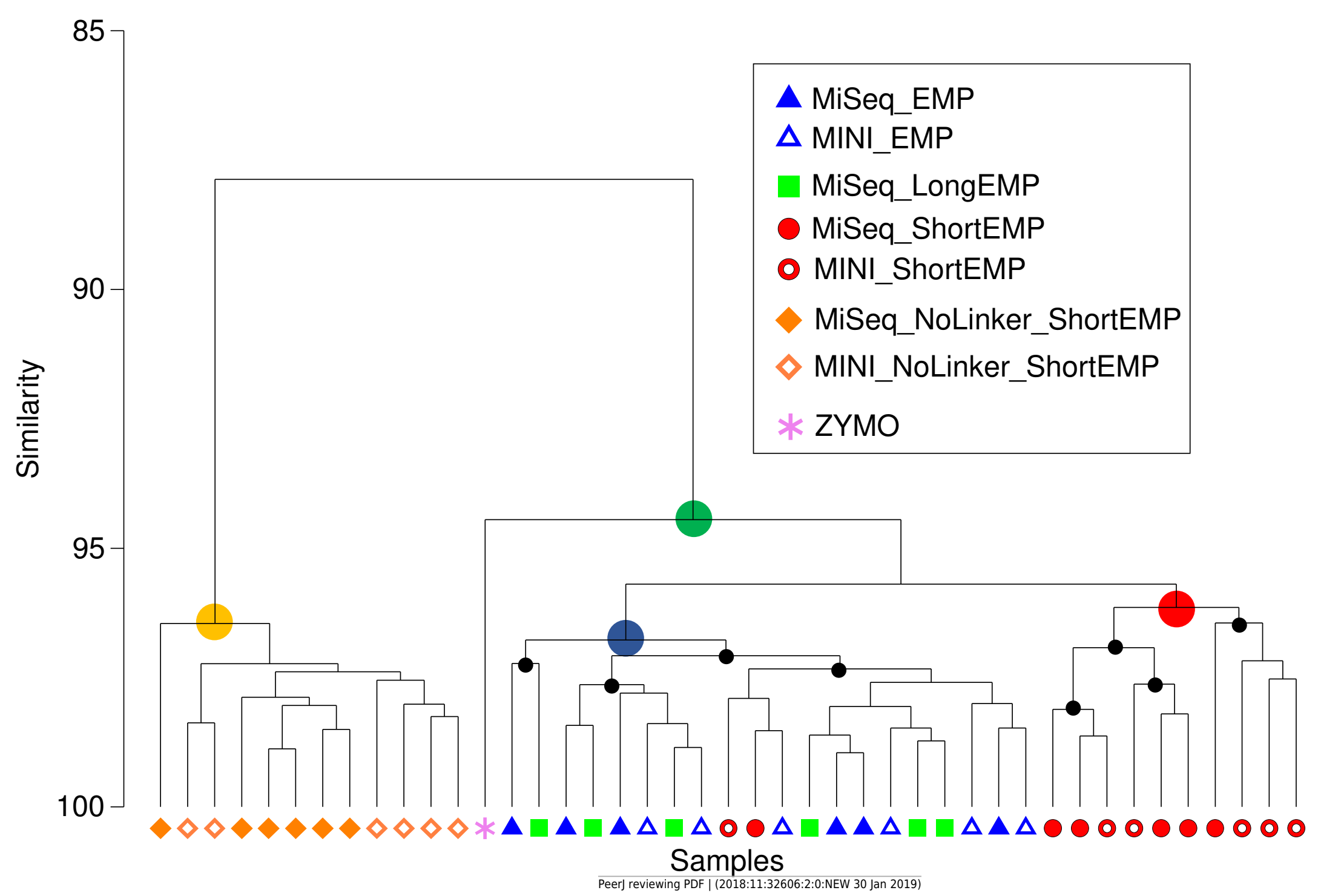




\section{Figure $\mathbf{5}$ (on next page)}

Effect of phiX spike-in on observed microbial communities with amplicons generated using ShortEMP primers

Genus-level annotations of sequence data generated on an Illumina MiniSeq instrument were visualized using mMDS ordination employing a distance matrix based on Bray-Curtis similarity. Rarefaction was performed to a depth of 30,000 sequences per sample. For each ShortEMP PCR condition, six technical replicates were analyzed using either $\sim 20 \%$ or $\sim 1 \%$ phiX spike-in. Small differences, consistent with run-to-run variation, were observed between the two sequencing runs (ANOSIM: $40^{\circ} \mathrm{C} R=0.146, p=0.052 ; 45^{\circ} \mathrm{C} R=0.394, p=0.011 ; 50^{\circ} \mathrm{C}$ $R=0.178, p=0.100$ ). Ellipses represent a $95 \%$ confidence interval around the centroid. 


\section{Table $\mathbf{1}$ (on next page)}

Alpha diversity indices of observed microbial communities

Shannon indices and richness were calculated at the taxonomic levels of genus and at the operational taxonomic unit (OTU97) level. For each primer set (EMP or ShortEMP), an average and standard deviation of six technical replicates is shown. Kruskal-Wallis tests (KW) were performed to determine if observed diversity was significantly different between EMP and ShortEMP analyses. Diversity indices were not significantly different between primer sets with the exception of OTU-level Shannon index for the Soil sample. 
Table 1

\begin{tabular}{|l|c|c|}
\hline \multicolumn{1}{|c|}{ Sample } & OTU Shannon & OTU Richness \\
\hline EMP_LMC_40 & $6.88 \pm 0.05$ & $3787.60 \pm 97.70$ \\
\hline EMP_LMC_45 & $6.81 \pm 0.09$ & $3738.80 \pm 81.14$ \\
\hline EMP_LMC_50 & $6.86 \pm 0.02$ & $3810.80 \pm 57.22$ \\
\hline ShortEMP_LMC_40 & $6.81 \pm 0.10$ & $3708.60 \pm 77.73$ \\
\hline ShortEMP_LMC_45 & $6.82 \pm 0.02$ & $3780.00 \pm 42.27$ \\
\hline ShortEMP_LMC_50 & $6.86 \pm 0.06$ & $3862.80 \pm 107.70$ \\
\hline \multicolumn{2}{|c|}{ KW } & $p=0.134$
\end{tabular}

\begin{tabular}{|l|c|c|}
\hline \multicolumn{1}{|c|}{ Sample } & Genus Shannon & Genus Richness \\
\hline EMP_LMC_40 & $4.76 \pm 0.02$ & $545.60 \pm 11.35$ \\
\hline EMP_LMC_45 & $4.74 \pm 0.06$ & $546.00 \pm 13.21$ \\
\hline EMP_LMC_50 & $4.77 \pm 0.02$ & $545.00 \pm 10.89$ \\
\hline ShortEMP_LMC_40 & $4.76 \pm 0.04$ & $534.60 \pm 10.78$ \\
\hline ShortEMP_LMC_45 & $4.73 \pm 0.01$ & $533.00 \pm 4.18$ \\
\hline ShortEMP_LMC_50 & $4.77 \pm 0.03$ & $542.20 \pm 5.81$ \\
\hline \multicolumn{2}{|c|}{ KW } & $p=0.107$
\end{tabular}

\begin{tabular}{|c|c|c|}
\hline EMP_Soil_50 & $7.16 \pm 0.03$ & $2907.50 \pm 99.02$ \\
\hline ShortEMP_Soil_45 & $7.09 \pm 0.04$ & $2832.67 \pm 65.56$ \\
\hline
\end{tabular}

\begin{tabular}{|c|c|c|}
\hline EMP_Soil_50 & $4.82 \pm 0.02$ & $414.50 \pm 10.45$ \\
\hline ShortEMP_Soil_45 & $4.80 \pm 0.03$ & $418.17 \pm 24.17$ \\
\hline
\end{tabular}

\begin{tabular}{|c|c|c|}
\hline EMP_Feces_50 & $4.94 \pm 0.04$ & $563.17 \pm 25.34$ \\
\hline ShortEMP_Feces_45 & $4.90 \pm 0.08$ & $605.17 \pm 65.49$ \\
\hline KW & $p=0.200$ & $\mathrm{p}=0.055$ \\
\hline
\end{tabular}

\begin{tabular}{|c|c|c|}
\hline EMP_Feces_50 & $2.41 \pm 0.00$ & $50.00 \pm 2.37$ \\
\hline ShortEMP_Feces_45 & $2.39 \pm 0.05$ & $52.50 \pm 2.51$ \\
\hline
\end{tabular}




\section{Table 2 (on next page)}

Ideal score calculations for analysis of the 'Zymo' Standard

The Ideal score is a summation of the absolute difference between the expected relative abundance and the observed relative abundance for each feature in a multi-feature dataset. Lower Ideal scores indicate a better representation of the expected community. KruskalWallis test (KW) p-values are shown for comparisons across temperatures or between EMP and ShortEMP primers at single temperatures. SD = standard deviation. 
Table 2

\begin{tabular}{|c|c|c|c|}
\hline $\begin{array}{c}\text { Annealing } \\
\text { Temp. }\end{array}$ & EMP (SD) & ShortEMP (SD) & KW \\
\hline $\mathbf{4 0}^{\circ} \mathbf{C}$ & $30.33(1.75)$ & $25.10(6.29)$ & 0.054 \\
\hline $\mathbf{4 5}^{\circ} \mathbf{C}$ & $16.03(1.54)$ & $12.95(0.98)$ & 0.006 \\
\hline $\mathbf{5 0}^{\circ} \mathbf{C}$ & $8.95(1.52)$ & $13.33(1.49)$ & 0.006 \\
\hline $\mathbf{K W}$ & 0.001 & 0.003 & \\
\hline
\end{tabular}

\begin{tabular}{|c|c|c|c|}
\cline { 2 - 4 } & EMP50 (SD) & ShortEMP45 (SD) & KW \\
\cline { 2 - 4 } $\begin{array}{c}\text { ShortEMP 45 } \\
\text { vs EMP } \mathbf{5 0}^{\circ} \mathbf{C}\end{array}$ & $8.95(1.52)$ & $12.95(0.98)$ & 0.006 \\
\cline { 2 - 4 } & & & \\
\end{tabular}

